\title{
New Microscopic Methods for the Functional Imaging of Energy Materials at the Nanoscale
}

\author{
Marina S. Leite ${ }^{1,2}$
}

1. Department of Materials Science and Engineering, University of Maryland, College Park, USA.

2. Institute for Research in Electronics and Applied Physics, University of Maryland, College Park, USA.

The replacement of fossil fuel-based technologies still requires an effective high-efficiency and low-cost photovoltaic (PV) technology. According to the National Renewable Energy Laboratory (NREL) Renewable Electricity Futures Study, $80 \%$ of the power generated in the US could be from renewable sources by 2050 [1], which requires that PV electricity generation increases to $>10 \%$. Yet, only $1 \%$ of the electricity is generated by solar power today [2]. The current high cost/Watt, $\$ 5.3 / \mathrm{W}$ for $<10 \mathrm{~kW}$ systems, is still the main challenge to achieve NREL's target. Perovskites and thin-film polycrystalline materials are a potential alternative for low-cost PV, but improvement in efficiency is required to ensure low cost/W. The performance of the best solar cells is still limited by the open-circuit voltage $\left(\mathrm{V}_{\mathrm{oc}}\right)$, the maximum voltage available from a PV device, which is related to the radiative recombination (photon emission) within the material. The $\mathrm{V}_{\mathrm{oc}}$ of both well-established and emerging materials is considerably below theoretical predictions [3]. This work addresses this critical limiting factor that constrains perovskite and thin-film polycrystalline solar cells' performance. While macroscopic light currentvoltage (I-V) measurements are useful for determining the overall device performance, a nanoscale imaging spectroscopy method to spatially resolve the local optical and electrical properties to access charge carrier recombination processes within materials for PV is crucial for understanding inefficiencies $[4,5]$.

Here, I present an imaging platform for mapping spatial variations in $\mathrm{V}_{\text {oc }}$ based on two techniques. First, we use confocal optical microscopy and spatially resolve the voltage within $\mathrm{Cu}(\mathrm{In}, \mathrm{Ga}) \mathrm{Se}_{2}$ (CIGS) grains, where we find variations $>20 \%$, likely related to their structural properties [6]. Further, we present a qualitative tomography of charge carrier recombination through spectrally dependent scans. The relevance of these measurements lies on establishing the correlation between the materials' structural properties and physical behavior.

To overcome the diffraction limit we realized a variant of illuminated Kelvin-probe force microscopy (KPFM) to map the local $\mathrm{V}_{\text {oc }}$ of materials for solar cells with nanoscale resolution $<50 \mathrm{~nm}$. By mapping the contact potential difference of half processed devices under dark and illuminated conditions, we quantify the $\mathrm{V}_{\mathrm{oc}}$ locally [7]. This nanoscale functional imaging paradigm works in ambient conditions and is nondestructive, enabling the diagnosis of how the different processing steps can influence the recombination within the material and, therefore, the ultimate performance of devices. We demonstrate its universality by imaging $\mathrm{V}_{\text {oc }}$ in GaAs, $\mathrm{Si}$, CdTe, and CIGS solar cells [7].

The understanding of the driving forces for degradation in hybrid organic-inorganic perovskites can transform the PV industry. While these materials present unprecedented potential for high-performance and low-cost photovoltaics, it changes upon exposure to humidity, oxygen, temperature, light, and bias. Thus, material degradation must be controlled at the length scale of the grains composing the perovskite thin films. We expand the $\mathrm{V}_{\text {oc }}$ method described above to probe the dynamic electrical response of perovskites. We realize fast-KPFM (16 s/scan) and map the real-time changes in voltage [8] that take 
place upon perovskites' illumination under 1-sun, in a low relative humidity environment. We measure spatial variations in local $\mathrm{V}_{\mathrm{oc}}>300 \mathrm{mV}$, and ion motion within one perovskite grain [8].

An overview of the different imaging methods currently applied to energy harvesting materials, ranging from near-field scanning optical microscopy [9] to photoluminescence microscopy will also be provided. In particular, the relevant considerations required to ensure that quantitative information about solar cells operation can be obtained, as well as all calibrations needed to compare nanoscale measurements with macroscopic device performance [4]. Finally, the need for 'big data' analytics will be presented [10].

\section{References:}

[1] http://www.nrel.gov/analysis/re_futures/

[2] US Energy Information Administration, Table 1.1.A. Net Generation by Other Renewable Sources: Total (All Sectors), 2003-November 2013, Table 1.1. Net Generation by Energy Source: Total (All Sectors), 2003-Nov201

[3] W Shockley and HJ Queisser, Journal of Applied Physics 32 (1961), p. 510.

[4] EM Tennyson, JM Howard, and MS Leite, ACS Energy Letters 2 (2017), p. 1825 - Invited Perspective.

[5] EM Tennyson, C Gong, and MS Leite, ACS Energy Letters 2 (2017), p. 2761 - Invited Review.

[6] EM Tennyson, et al, ACS Energy Letters 1 (2016), p. 899.

[7] EM Tennyson, et al, Adv. Energy Materials 5 (2015), p. 1501142.

[8] JL Garrett, et al, Nano Letters 17 (2017), p. 2554.

[9] MS Leite, et al., ACS Nano 8 (2014), p. 11883.

[10] The author acknowledges the financial support from NSF-ECCS (16-10833), the 2016 American Physical Society Ovshinsky Sustainability Fellowship, the 2015 RASA/UMD award.

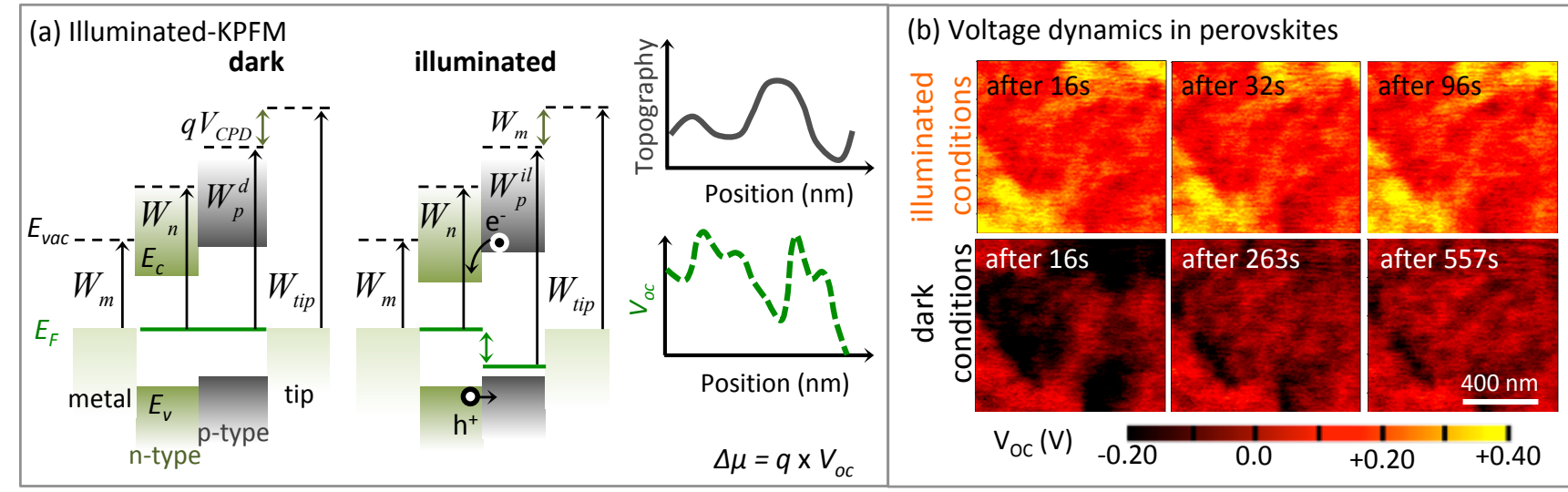

Figure 1. (a) Concept of illuminated-KPFM realized to map open-circuit voltage $\left(V_{o c}\right)$ with spatial resolution $<50 \mathrm{~nm}$. (b) Dark- and illuminated-KPFM showing real-time changes in the local voltage of perovskites with nanoscale spatial resolution. 